\title{
Anaplastic Lymphoma Kinase Gene Analysis as a Useful Tool for Identifying Primary Unknown Metastatic Lung Adenocarcinoma
}

\author{
Naoki Watanabe, Tomoya Ishii, Takayuki Takahama, Akira Tadokoro, Nobuhiro Kanaji, \\ Hiroaki Dobashi and Shuji Bandoh
}

\begin{abstract}
A 55-year-old woman was admitted for an evaluation of a mediastinal mass, bilateral cervical lymphadenopathy and a left breast tumor. Although pathology revealed a diagnosis of breast cancer, the cervical lymph nodes differed from the breast lesion. An anaplastic lymphoma kinase $(A L K)$ gene analysis revealed $A L K$ rearrangement in the cervical lymph nodes only, which were therefore diagnosed as reflective of metastasis of lung adenocarcinoma. The mediastinal tumor was also diagnosed as an ALK-positive lung adenocarcinoma based on its therapeutic response. $A L K$ gene analyses can be used to identify primary lesions in patients with cancers of unknown primary sites.
\end{abstract}

Key words: cancer of unknown primary site (CUP), mediastinal type lung cancer, $A L K$ gene analysis, adenocarcinoma

(Intern Med 53: 2711-2715, 2014)

(DOI: 10.2169/internalmedicine.53.2775)

\section{Introduction}

Cancer of unknown primary site (CUP) involves the documentation of a metastatic cancer in the absence of an identifiable primary lesion following the application of a standard diagnostic approach. Depending on the definition of CUP and population of patients studied, $1-5 \%$ of cancer patients are diagnosed with this clinical entity $(1,2)$. Identifying the primary lesion is essential for selecting appropriate locoregional and/or chemotherapy regimens.

Assessments of patients with suspected metastasis of malignancy should include a complete medical history, physical examination, imaging examinations and laboratory, histopathological and immunohistochemical studies (3). Although several gene expression-profiling assays have recently been developed to determine the primary site of metastatic squamous cell carcinoma of the head and neck $(4,5)$, no such tests have been reported for lung cancer to date.

Case Report
A 55-year-old woman who presented to an otolaryngolo-
gist with a three-month history of coughing and hoarseness
was diagnosed with left recurrent nerve paralysis. She had
no notable medical history or smoking habits. Computed to-
mography (CT) of the neck and chest revealed a mediastinal
mass under the aortic arch, with bilateral cervical lymphade-
nopathy and a left breast tumor (Fig. 1). However, radiology
showed no evidence of a primary site, including the lung
field. The distribution of cervical lymph nodes and presence
of a mediastinal mass were atypical for metastases of breast
cancer; therefore, CUP was diagnosed at the time of the ini-
tial examination. The serum carcinoembryonic antigen
(CEA) level was 74.8 ng/mL (reference range: $0-5.0$ ), and a
chest X-ray and urinalysis showed no abnormal findings. Al-
though positron emission tomography (PET) demonstrated
fluorodeoxyglucose (FDG) accumulation in all CT-identified
lesions (Fig. 1), the primary lesion remained unknown.

Department of Internal Medicine, Division of Endocrinology and Metabolism, Hematology, Rheumatology and Respiratory Medicine, Faculty of Medicine, Kagawa University, Japan

Received for publication February 26, 2014; Accepted for publication May 21, 2014

Correspondence to Dr. Naoki Watanabe, naoki-w@med.kagawa-u.ac.jp 

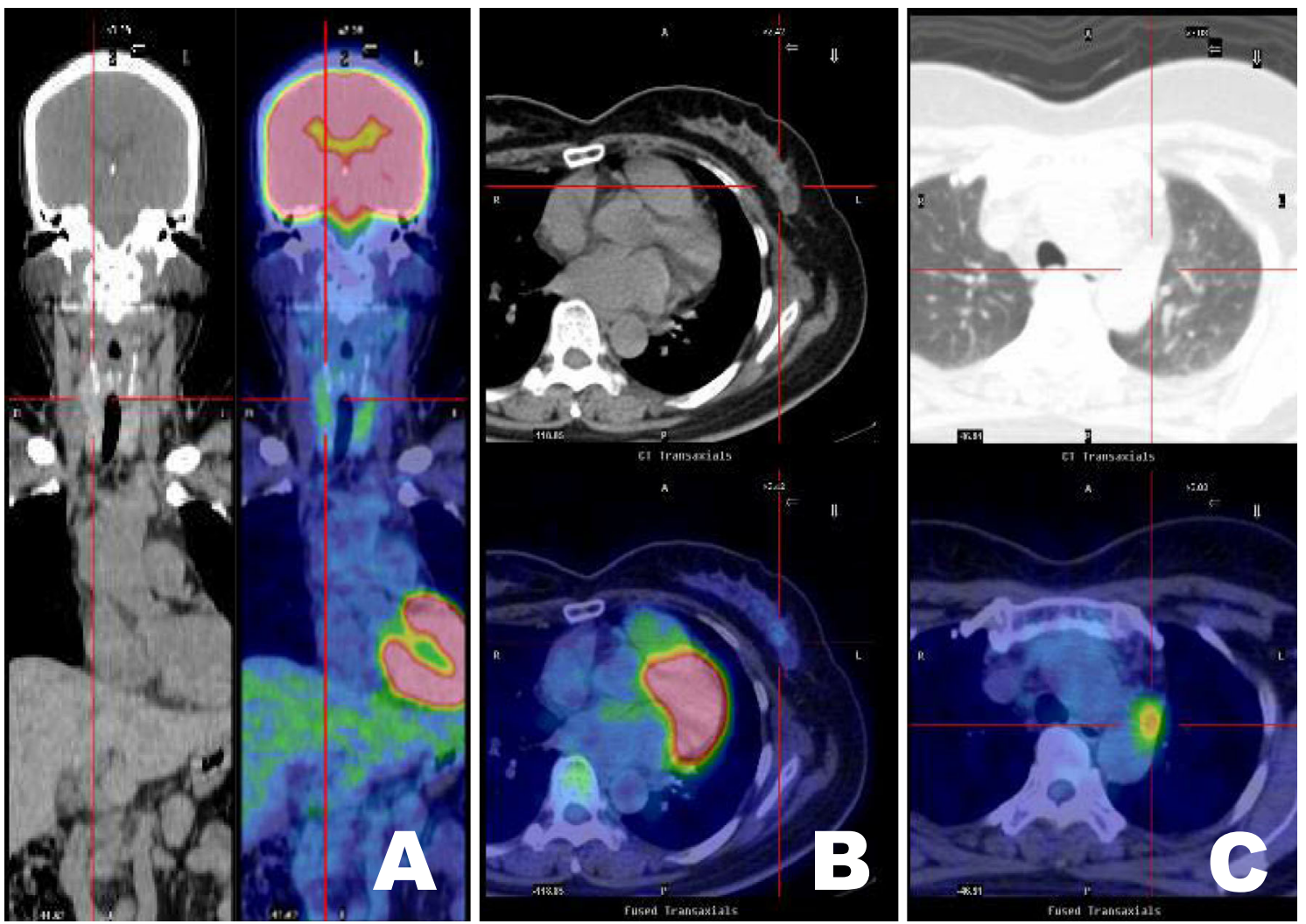

Figure 1. Neck and chest PET/CT findings. (A) Bilateral cervical lymphadenopathy; (B) Left breast tumor; (C) Mediastinal mass under the aortic arch.

In order to identify the primary lesion, we pathologically examined two specimens obtained from the breast mass and cervical lymph node. Taking into consideration the degree of invasiveness, a mediastinal lymph node biopsy was not performed. Hematoxylin and Eosin (H\&E) staining showed adenocarcinoma in both specimens, and adenocarcinoma breast tumor cells were found to have proliferated intraductally (Fig. 2A). According to the immunohistochemical (IHC) analysis, the breast tumor was positive for human epidermal growth factor receptor type 2 (HER2) and gross cystic disease fluid protein-15 (GCDFP-15), but not mammaglobin, estrogen receptor (ER) or progesterone receptor (PgR). The breast tumor was diagnosed as breast cancer based on these results. However, the cervical lymph node did not stain positive for any of the breast cancer markers mentioned above (Fig. 2C). In addition, the breast tumor specimens were negative for thyroid transcription factor-1 (TTF-1), napsin-A and surfactant protein, lung cancer markers. On the other hand, the cervical lymph nodes were positive for these markers in only 5\% of tumor cells. The cervical lymph node lesion was therefore thought to have a different origin from the breast lesion. We also conducted advanced analyses of epidermal growth factor receptor $(E G F R)$ gene and anaplastic lymphoma kinase $(A L K)$ gene, driver genes for lung adenocarcinoma. Both of the specimens exhibited wild-type $E G F R$ on reverse transcriptase-polymerase chain reaction (RT-PCR), as previously described (6). Interestingly, only the cervical lymph node was positive for ALK on both IHC (Fig. 2B, D) and fluorescence in situ hybridization (FISH) (Fig. 3). The cervical lymph node was therefore diagnosed as a metastasis of stage IV lung adenocarcinoma, although the primary lesion was absent in the lung field. Meanwhile, the breast lesion was judged to be at stage I.

Under a diagnosis of CUP on admission, we initiated treatment with chemotherapy with carboplatin (AUC 5) and docetaxel $\left(60 \mathrm{mg} / \mathrm{m}^{2}\right)$. Following the administration of the first course of chemotherapy, the patient was estimated to have stable disease. Additionally, the serum CEA level increased to $80.4 \mathrm{ng} / \mathrm{mL}$, in spite of this treatment. The presence of ALK-positive lung adenocarcinoma was confirmed after chemotherapy, and we therefore started therapy with crizotinib (500 mg daily)- an ALK tyrosine kinase inhibitor (TKI)- as the second-line treatment, which had a cytoreductive effect on both the cervical lymph node metastasis and mediastinal tumor (Fig. 4), further indicating that the mediastinal mass was ALK-positive lung adenocarcinoma. Moreover, the serum CEA level remarkably decreased to $8.9 \mathrm{ng} /$ $\mathrm{mL}$ after three months of crizotinib therapy; however, the breast lesion exhibited no reductions in size. Since breast cancer of stage I does not influence the prognosis, as compared with advanced lung cancer, we are currently observing the patient's course in consultation with a breast surgeon. 


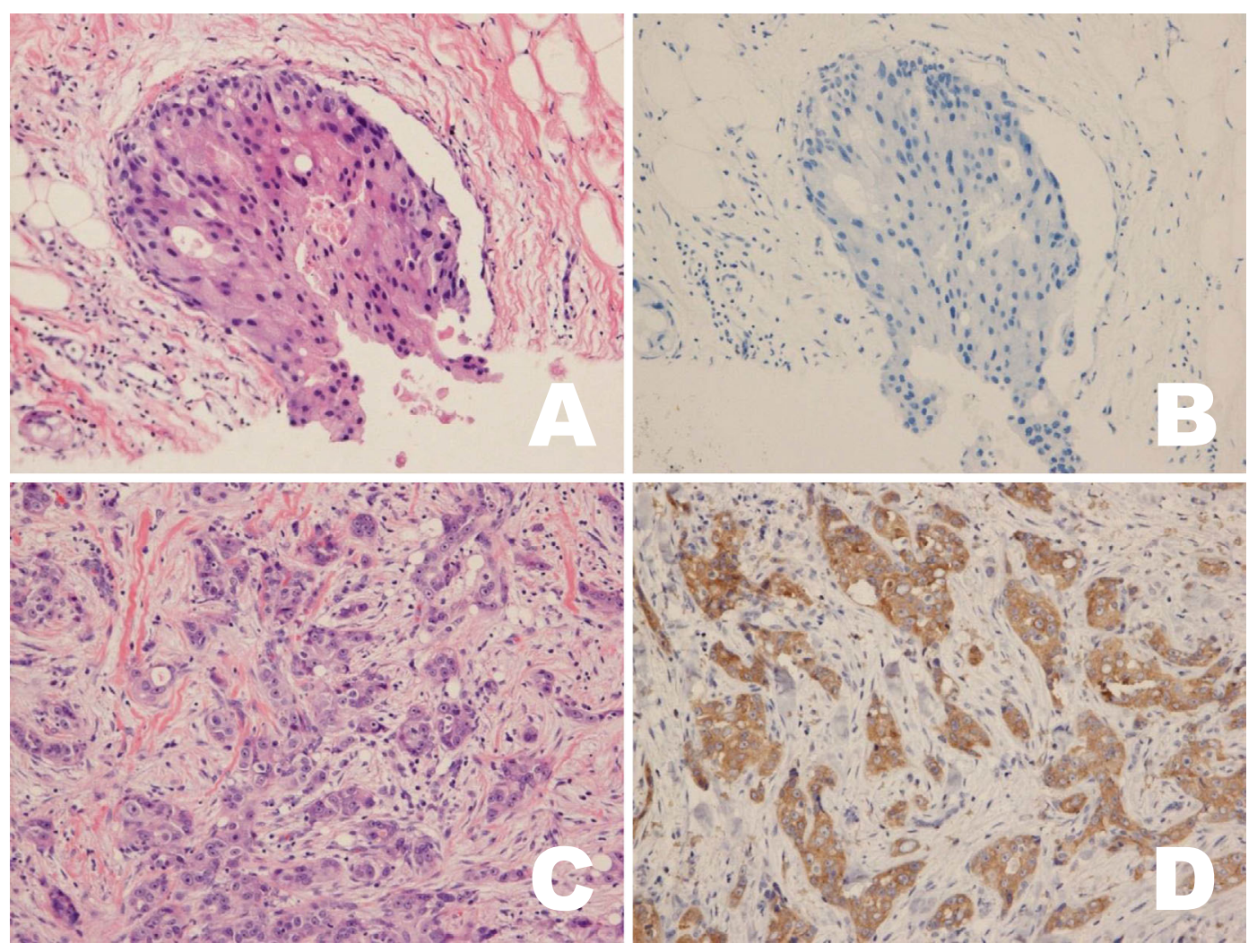

Figure 2. Microscopic findings of the breast tumor and cervical lymph node. (A) Hematoxylin and Eosin (H\&E) staining shows adenocarcinoma in the mammary duct (original magnification $\times 100$ ); (B) Immunohistochemistry (IHC) of ALK shows no staining in the breast tumor cells (original magnification $\times 100$ ); (C) H\&E staining shows the cervical lymph node to be infiltrated with adenocarcinoma (original magnification $\times 100$ ); (D) IHC of the ALK-positive tumor cells in the cervical lymph node (original magnification $\times 100$; positive areas appear brown).

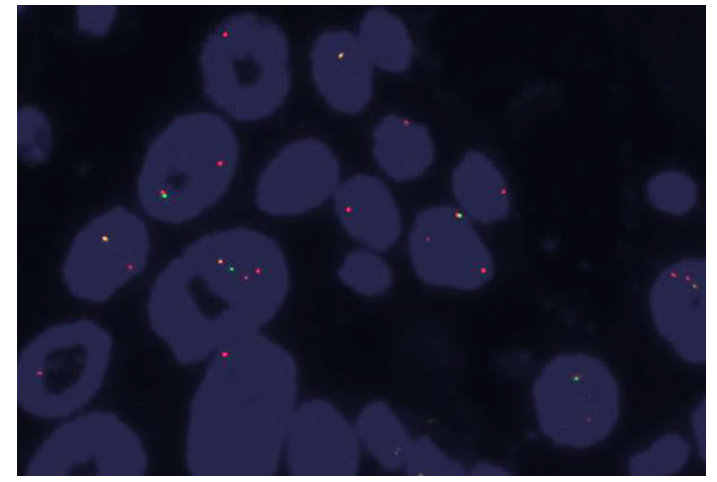

Figure 3. FISH study demonstrating $A L K$ rearrangement. $A L K$ break-apart probes were used to detect the rearrangement of this gene. The red, green and yellow signals represent 3' $A L K$, 5' $A L K$ and 3'/5' $A L K$, respectively. The split between the red and green signals indicates the rearrangement of the ALK gene.

\section{Discussion}

Patients with CUP generally have a limited life expectancy, with a median survival of approximately six to nine months (7). However, some subsets have better prognoses and longer survival outcomes (8); therefore, the most important approach to treating CUP is to identify candidates for primary-specific therapy. In order to identify the primary lesion, optical microscopy of the biopsy specimens should first be performed. However, if $\mathrm{H} \& \mathrm{E}$ staining is insufficient to identify the primary lesion, IHC may be used to detect the origin of the tumor (9). Such analyses are useful in differentiating lesions and determining the histological and pathological diagnoses (3). The most common histology is adenocarcinoma, for which the seven most frequent primary sites are the colon, breast, ovaries, lungs, stomach, pancreas and bile ducts.

Various IHC markers can also be used to identify the primary lesion. In cases of breast cancer, GCDFP-15 is $34.9 \%$ specific and $98.8 \%$ sensitive, while CK-7 is $90.7 \%$ specific and $26.1 \%$ sensitive and ER is $67.4 \%$ specific and $96.8 \%$ sensitive. Meanwhile, in cases of lung cancer, TTF-1 is $88 \%$ specific and $100 \%$ sensitive and CK-7 is $100 \%$ specific and $28.5 \%$ sensitive (10). Stoll et al. reported that napsin-A is a useful surrogate marker of poorly differentiated lung adenocarcinoma or CUP, with a specificity of $96 \%$ and sensitivity of $65 \%$ for lung adenocarcinoma (11). In the present study, we were able to diagnose the patient's breast tumor as breast cancer based on immunostaining (HER2 and GCDFP15), whereas the cervical lymph node lesion was distin- 


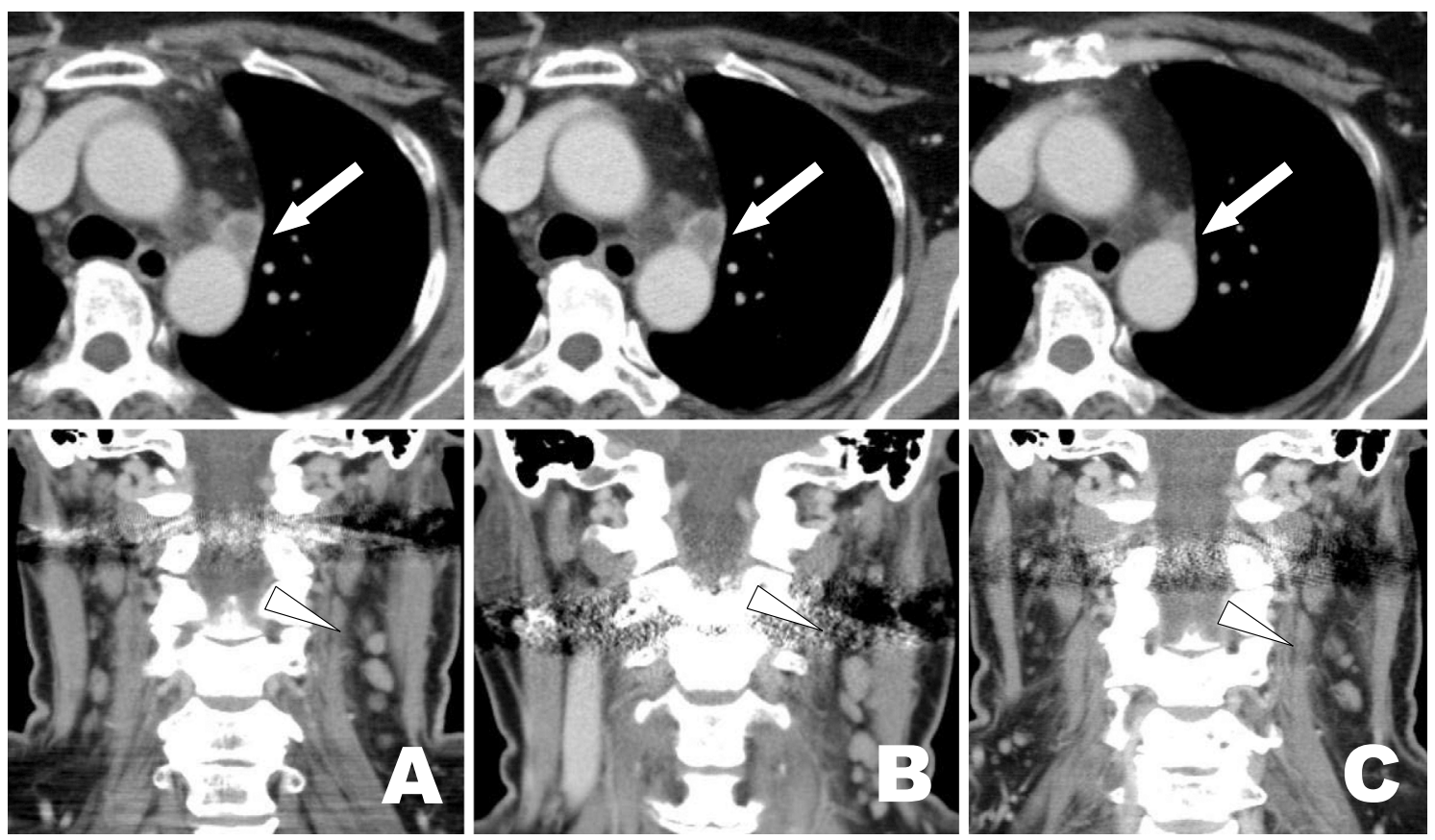

Figure 4. Chest CT findings before and after treatment with crizotinib. (A) Mediastinal mass shadow on admission; (B) After chemotherapy with carboplatin and docetaxel; (C) After one month of treatment with crizotinib. The arrows indicate the mediastinal mass and the arrowheads indicate the cervical lymph node.

guished from the breast lesion according to differences on IHC staining. Because the cervical lymph node specimen was positive for TTF-1, napsin-A and CK-7 in only 5\% of tumor cells, there was little evidence to suggest a diagnosis of lung cancer. Therefore, it was necessary to use a method for determining the primary lesion other than IHC.

Several chromosome rearrangements and fusion genes have been identified in patients with malignant tumors, and appropriate targeted therapies have been developed (12). The detection of chromosomal rearrangements and/or fusion genes is reportedly useful for both diagnosis and treatment in cases of sarcoma of the bone and soft tissue (13). Although no genetic and/or chromosomal analyses have been developed to identify lung cancer as a primary lesion, several driver genes (including EGFR, KRAS and $A L K$ ) have been detected in patients with lung cancer. Erlotinib and gefitinib are examples of EGFR TKIs that suppress signaling pathways, and thereby improve response rates, in selected patients with non-small cell lung cancer (NSCLC). In addition, crizotinib (Xalkori ${ }^{\circledR}$, Pfizer, New York, USA) is the first FDA-approved ALK TKI shown to be effective against advanced NSCLC associated with activated ALK kinase $(14,15)$. As these TKIs are effective in treating systemic metastatic lesions, the detection of driver genes may contribute to both diagnosing lung cancer and selecting the proper treatment strategy.

Three main methods are used to identify $A L K$ rearrangement: FISH, IHC and RT-PCR. Although the current standard method for detecting ALK-positive NSCLC is FISH, such analyses occasionally provide false-positive results, subsequently requiring testing with another diagnostic method, such as IHC staining, in order to further assess the efficacy of ALK inhibitors (16). Ideally, the diagnosis of $A L K$ rearrangement should be demonstrated using more than two methods. In the present case, $A L K$ rearrangement was detected according to both IHC and FISH (the break-apart method), which confirmed $A L K$ to be truly positive.

Activating mutations and translocations of the $A L K$ gene have been identified in several types of cancer, including anaplastic large-cell lymphoma, neuroblastoma and inflammatory myofibroblastic tumors other than NSCLC. In a few cases, ALK-positive colorectal cancers and breast cancers have also been reported (17). We therefore performed upper gastrointestinal endoscopy and colonoscopy in order to confirm the absence of colorectal, esophageal or gastric cancers.

Approximately $80 \%$ of patients with CUP belong to prognostically unfavorable subsets, which may not have standard treatments. Data obtained from non-randomized studies over the past 40 years have shown that the introduction of platinum or platinum-taxane combinations is associated with improved response rates and overall survival. In the present case, treatment with platinum-based chemotherapy was started as the initial therapy for due to the patient's poor prognosis, although crizotinib was later administered due to the clinical diagnosis of ALK-positive lung adenocarcinoma. If the results of the driver gene analysis had not been available, then it is unlikely that the patient would have received cancer type-specific treatment.

We conclude that driver gene analyses of lung adenocarcinoma are an important basic means of both identifying primary lesions and selecting appropriate molecular targeted therapy in patients with CUP. 
The authors state that they have no Conflict of Interest (COI).

\section{References}

1. Muir C. Cancer of unknown primary site. Cancer 75: 353-356, 1995.

2. Abbruzzese JL, Abbruzzese MC, Hess KR, Raber MN, Lenzi R, Frost P. Unknown primary carcinoma: natural history and prognostic factors in 657 consecutive patients. J Clin Oncol 12: 12721280, 1994.

3. National Comprehensive Cancer Network. NCCN Clinical Practice Guidelines in Oncology [Internet]. [cited 2013 Dec 1]. Available from: http://www.nccn.org/professionals/physician_gls/pdf/occult. pdf

4. Zhang MQ, El-Mofty SK, Davila RM. Detection of human papillomavirus-related squamous cell carcinoma cytologically and by in situ hybridization in fine-needle aspiration biopsies of cervical metastasis: a tool for identifying the site of an occult head and neck primary. Cancer 114: 118-123, 2008.

5. Califano J, Westra WH, Koch W, et al. Unknown primary head and neck squamous cell carcinoma: molecular identification of the site of origin. J Natl Cancer Inst 91: 599-604, 1999.

6. Kanaji N, Bandoh S, Ishii T, et al. Detection of epidermal growth factor receptor mutation in a few cancer cells from transbronchial cytologic specimens by reverse transcriptase-polymerase chain reaction. Mol Diagn Ther 15: 353-359, 2011.

7. Pavlidis N, Briasoulis E, Hainsworth J, Greco FA. Diagnostic and therapeutic management of cancer of an unknown primary. Eur J Cancer 39: 1990-2005, 2003.
8. Pavlidis N, Pentheroudakis G. Cancer of unknown primary site. Lancet 379: 1428-1435, 2012.

9. Jaffer S, Bleiweiss IJ. Beyond hematoxylin and eosin: the role of immunohistochemistry in surgical pathology. Cancer Invest 22: 445-465, 2004.

10. Park SY, Kim BH, Kim JH, Lee S, Kang GH. Panels of immunohistochemical markers help determine primary sites of metastatic adenocarcinoma. Arch Pathol Lab Med 131: 1561-1567, 2007.

11. Stoll LM, Johnson MW, Gabrielson E, Askin F, Clark DP, Li QK. The utility of Napsin-A in the identification of primary and metastatic lung adenocarcinoma among cytologically poorly differentiated carcinomas. Cancer Cytopathol 118: 441-449, 2010.

12. National Cancer Institute. National Cancer Institute, Factsheet [Internet]. [cited 2013 Dec 1]. Available from: http://www.cancer.gov/ cancertopics/factsheet/Therapy/targeted

13. Antonescu CR. The role of genetic testing in soft tissue sarcoma. Histopathology 48: 13-21, 2006.

14. Lynch TJ, Bell DW, Sordella R, et al. Activating mutations in the epidermal growth factor receptor underlying responsiveness of non-small-cell lung cancer to gefitinib. N Engl J Med 350: 21292139, 2004.

15. Kwak EL, Bang Y-J, Camidge DR, et al. Anaplastic lymphoma kinase inhibition in non-small-cell lung cancer. $\mathrm{N}$ Engl $\mathrm{J}$ Med 363: 1693-1703, 2010.

16. Chihara D, Suzuki R. More on crizotinib. N Engl J Med 364: 776-777; author reply 778, 2011.

17. Roskoski R. Jr. Anaplastic lymphoma kinase (ALK): structure, oncogenic activation, and pharmacological inhibition. Pharmacological Res 68: 68-94, 2013.

(C) 2014 The Japanese Society of Internal Medicine http://www.naika.or.jp/imonline/index.html 